\title{
Centralized Resource Allocation and Target Setting Based on Data Envelopment Analysis Model
}

\author{
Tiantan Yang $\mathbb{D},{ }^{1}$ Pingchun Wang $\mathbb{D}^{2},{ }^{2}$ and Feng $\mathrm{Li} \mathbb{D}^{3}$ \\ ${ }^{1}$ Department of Philosophy of Science, University of Science and Technology of China, Hefei, Anhui Province 230026, China \\ ${ }^{2}$ School of Management, University of Science and Technology of China, Hefei, Anhui Province 230026, China \\ ${ }^{3}$ School of Business Administration, Southwestern University of Finance and Economics, Chengdu 611130, China \\ Correspondence should be addressed to Feng Li; lfeng90@mail.ustc.edu.cn
}

Received 17 August 2017; Accepted 31 December 2017; Published 5 February 2018

Academic Editor: Josefa Mula

Copyright (C) 2018 Tiantan Yang et al. This is an open access article distributed under the Creative Commons Attribution License, which permits unrestricted use, distribution, and reproduction in any medium, provided the original work is properly cited.

\begin{abstract}
This paper aims to develop a data envelopment analysis (DEA) based model for allocating input resources and deciding output targets in organizations with a centralized decision-making environment, for example, banks, police stations, and supermarket chains. The central decision-maker has an interest in maximizing the total output production and at the same time minimizing the total input consumption. Traditionally, all decision-making units (DMUs) can be easily projected to the efficient frontier, which is a mathematical feasibility; however, it does not guarantee the managerial feasibility during the planning period. In this paper, we will take potential limitations of input-output changes into account by building a difficulty coefficient matrix of modifying their production in the current production possibility set so that the solution guarantees managerial feasibilities. Three objectives, namely, maximizing aggregated outputs, minimizing the consumption of input resources, and minimizing the total difficulty coefficient, are proposed and incorporated into the formation of resource allocation and target setting scheme. Building on this, we combine DEA and multiobjective programming to solve the resource allocation and target setting problem. In the end, we apply our proposed approach to a real-world problem of sixteen chain hotels to illustrate the efficacy and usefulness of the proposed approach.
\end{abstract}

\section{Introduction}

In many real scenarios, there are many complex organizations typically consisting of a number of individual production units, such as an education authority that may correspond to different schools. In such organizational environment, all the decision-making units (DMUs) fall under the umbrella of a central unit which has power to oversee them. Management of these large companies and public service organizations needs coordinating mechanism to better manage its resources to fulfill its mission. How to allocate resource and set targets is an important thing for the central unit. In this research we deal with the problem of allocating input resources and setting output targets which happen on the organizational level. The organization will have to set targets for each unit which should be logically linked to the resource that organization allocates to each unit simultaneously.

Resource allocation and/or target setting is a typical problem in organizations, which has become one of hottest topics and classical applications in economic and managerial field. Data envelopment analysis (DEA) has proved to be a powerful tool for determining the relative efficiencies of the decision-making units, which consume multiple inputs to produce multiple outputs. Since DEA was first introduced by Charnes et al. [1], it has been prevalent to deal with complex problems and cases in the public or private organization. Although the original object of this method is on the efficiency measurement, it has been applied to various activities [2-4]. Additionally, it has recently been developed for resource allocation and target setting. Cook and Kress [5] made the first attempt to allocate a total fixed cost among a set of DMUs based on two principles of efficiency invariance and Pareto optimality. Jahanshahloo et al. $[6,7]$ showed that the principles used in Cook and Kress [5] might be violated. Beasley [8] used the concept of cross-efficiency to maximize the average efficiency score of all DMUs for allocating fixed costs under a set of common weights. Subsequently, Fang and Zhang [9] formulated a 
bicriteria DEA-based resource allocation model. The first objective was to maximize the average DMU efficiency (the total efficiency at the organization level) as same as the model of Beasley [8]. The second objective represented the minimal quantity among all the efficiency variables (equity among the individual unit's efficiency). Lin and Chen [10] allocated a fixed input based on super-CCR efficiency and efficiency invariance principle. Yu et al. [11] allocated a fixed cost using two-stage network data envelopment approach. $\mathrm{Li}$ et al. [12] proposed a game-DEA cross-efficiency approach for allocating a fixed cost. Golany [13] used a multiobjective programming to determine output goals based on DEA models. Athanassopoulos [14] has presented a Go-DEA model for dealing with the conflicting objection of efficiency, effectiveness, and quality in resource allocation. Furthermore, Athanassopoulos [15] proposed a TARBA model based on a similar framework to that of Go-DEA, which will be solved in two steps. First, the optimal weights of each DMU are determined using a multiplier DEA model. Then the optimal weights are used to define feasible trade-offs in resource allocation. Matin and Azizi [16] addressed the target setting problem with negative data. Dehnokhalaji et al. [17] addressed the resource allocation problem by keeping the cost efficiencies improved or unchanged. In that paper, two strategies are considered, namely, keeping the cost efficiencies of all DMUs unchanged by fixing the technical and allocative efficiencies and improving the cost efficiencies of DMUs after the allocation as much as possible.

Normally, the output target is set simultaneously by allocating some input resources. Amirteimoori and Kordrostami [18] proposed a resource allocation and target setting model by combining the efficiency invariance principle of Cook and Kress [5] and the common weight of Beasley [8]. Amirteimoori and Tabar [19] proposed a DEA model which simultaneously allocates fixed resource and sets output targets. In their paper, the authors introduced goals achievement variables for the efficiency level, allocated resources, and set targets to obtain a unique allocation plan. By considering the internal structure, Bi et al. [20] addressed the problem of resource allocation and target setting for a parallel production system. Lin [21] proposed a method for allocating input resources and setting output targets in such a way that the efficiency invariance principle was satisfied. Lotfi et al. [22] and Hatami-Marbini et al. [23] combined goal programming method with a set of common weights to address the resource allocation and target setting problem. Further, the postallocation efficiency scores were maximized. Recently, Li et al. [24] proposed a new DEA-based approach for resource allocation and target setting based on common weights and efficiency invariance. Their approach allows the trade-off between common weights and efficiency invariance principles, and the final allocation and target scheme is determined by minimizing the distance of two possible plans.

In many real managerial applications, there are situations where all DMUs are controlled by a centralized decisionmaker. Under the circumstances, the centralized decisionmaker would like to increase the overall output production and decrease the total input resource consumption. Lozano and Villa [25] set up the centralized data envelopment analysis models to minimize the overall resource consumption or to maximize the total output production in an organization, rather than considering each DMU separately. They formulated two models for such resource allocation problem. One type of models seeks radial reductions of the total consumption of every input while the other model seeks separate reductions for each input. Lozano and Villa [26] extended the work of Lozano and Villa [25] to more general multiple-input-multiple-output centralized cases with both radial and nonradial models. Lozano et al. [27] also used the centralized approach to analyze the performance of a number of Spanish municipalities in terms of the amount of glass recycled and counted an optimal allocation of collecting boxes for maximizing the recycling of glass waste; in addition, they also have taken integer variables into account while using the centralized way. Moreover, Asmild et al. [28] extended and modified the model of Lozano et al. [27], proposing the centralized resource allocation and target setting model which only considers adjustments of the previous inefficiency units. Korhonen and Syrjänen [29] developed an interactive formal approach based on DEA and multiple objective linear programming in organizations with a centralized decision-making environment and applied it to solve a resource allocation problem in order to maximize the total production under the assumption that the units can modify their current production possibility set. Pachkova [30] studied a restricted resource reallocation problem, where the restriction is formulated as the maximum cost the managers are willing to pay. Further, the author made a tradeoff between the highest efficiency the aggregated unit can achieve and the maximum allowed reallocation cost. Lozano et al. [31] reallocated the emission permits using three lexicographical objectives, namely, maximizing aggregated desirable production, minimizing undesirable total emissions, and minimizing the consumption of input resources. Given a capital budget constraint, Lozano et al. [32] proposed a series of centralized DEA models for individual and collective output target setting, input reallocation, and additional input acquisitions. Lozano [33] proposed a slack-based measure model for fixed cost and common revenue allocation under a centralized environment. Fang [34] proposed a new centralized approach for resource allocation based on efficiency analysis, in which a sequence of intermediate benchmark targets was determined as well as a level-wise improvement path, which can improve the DMUs to their ultimate targets on the efficient frontier in an implementable and realistic manner. Fang [35] proposed a centralized resource allocation approach based on revenue efficiency, and the allocation plan was determined by maximizing the total output revenue.

Traditionally, the DEA literature thinks that the unit is able to reach any point on the efficient frontier easily, which is a mathematical feasibility; however, it does not guarantee managerial feasibility during the planning period. Moreover, it projects on the efficiency frontier, which declares that the DMU has the potential to achieve the input reducing and output increasing, which provide a direction of each DMU's improvement. If the center decision-maker does not consider the effect of the restricted input reducing and output increasing, he will make incorrect decisions. In this text 
we will consider the potential restrictions of the change in the unit's production during the planning period when the center decision-maker faces the resource allocation and target setting problem. To this end, we build a difficulty coefficient matrix of modifying the current production so that the solution guarantees managerial feasibilities. Further, three objectives are used to generate the final resource allocation and target setting plan, namely, maximizing aggregated output, minimizing the consumption of input resources, and minimizing the total difficulty coefficient. The DEA methodology and multiobjective programming are combined to solve the problem.

The remainder of this paper is organized as follows: in Section 2, we revisit the preliminary work in DEA literature. Then in Section 3, we propose the new resource allocation and target setting approach. An empirical application was applied to illustrate the proposed approach in Section 4. Finally, we conclude this paper in Section 5.

\section{Preliminary Consideration}

At the starting point, we assume there exist $n$ DMUs, with each DMU consuming $m$ inputs and producing $s$ output. Now we denote $X_{j}=\left(x_{1 j}, \ldots, x_{m j}\right)$ and $Y_{j}=\left(y_{1 j}, \ldots, y_{s j}\right)$ as the vector of inputs and outputs of $\mathrm{DMU}_{j}(j=1, \ldots, n)$, respectively, where $x_{i j}$ and $y_{r j}$ are the observed levels of the $i$ th input and $r$ th output of DMU ${ }_{j}$. The basic DEA model, called CCR model, which is based on the constant returns to scale for assessing the relative efficiency of a given $\mathrm{DMU}_{0}$ has been proposed by Charnes et al. [1]. The basic model is presented as

$$
\begin{aligned}
e_{0}^{*}=\max & \frac{\sum_{r=1}^{s} \mu_{r} y_{r 0}}{\sum_{i=1}^{m} v_{i} x_{i 0}} \\
\text { s.t. } & \frac{\sum_{r=1}^{s} \mu_{r} y_{r j}}{\sum_{i=1}^{m} v_{i} x_{i j}} \leq 1, \quad j=1, \ldots, n \\
& \mu_{r}, v_{i} \geq \varepsilon, \quad r=1, \ldots, s, \quad i=1, \ldots, m .
\end{aligned}
$$

In the above model, $\mu_{r}(r=1, \ldots, s)$ and $v_{i}(i=1, \ldots, m)$ are unknown relative weights attached to the $r$ th output and $i$ th input, respectively. $\varepsilon$ presents a positive non-Archimedean infinitesimal number. It is clear that model (1) is a nonlinear model which is also called the multiplier model. We can easily transform model (1) to a liner equivalent as shown in the following model:

$$
\begin{aligned}
e_{0}^{*}=\max & \sum_{r=1}^{s} u_{r} y_{r 0} \\
\text { s.t. } & \sum_{r=1}^{s} u_{r} y_{r j}-\sum_{i=1}^{m} v_{i} x_{i j} \leq 0, \quad j=1, \ldots, n \\
& \sum_{i=1}^{m} v_{i} x_{i 0}=1 \\
& u_{r}, v_{i} \geq \varepsilon, \quad r=1, \ldots, s, \quad i=1, \ldots, m .
\end{aligned}
$$

Model (2) is an input-oriented model, which can be easily solved to calculate the optimal efficiency. $\mathrm{DMU}_{0}$ is defined as efficient if there exists at least one optimal $\left(u_{r}^{*}, \forall r ; v_{i}^{*}, \forall i\right)$ of model (2) and $\sum_{r=1}^{s} u_{r}^{*} y_{r 0}=1$ with $u_{r}^{*} \geq \varepsilon$ and $v_{i}^{*} \geq \varepsilon$. Otherwise $\mathrm{DMU}_{0}$ is inefficient.

Its dual problem is also useful in realistic applications, which is expressed as follows:

$$
\begin{aligned}
\theta_{0}^{*}=\max & \theta \\
\text { s.t. } & \sum_{j=1}^{n} \lambda_{j} x_{i j} \leq \theta x_{i o}, \quad i=1, \ldots, m \\
& \sum_{j=1}^{n} \lambda_{j} y_{r j} \geq y_{r o}, \quad r=1, \ldots, s \\
& \lambda_{j} \geq 0, \quad j=1, \ldots, n .
\end{aligned}
$$

The optimal objective function of model (3) ranges between zero and one. If the model receives a score of one, then $\mathrm{DMU}_{0}$ is defined as DEA efficient and DEA inefficient otherwise.

\section{DEA-Based Resource Allocation and Target Setting Model}

Traditional resource allocation and target setting model based on DEA methodology considers one unit at a time in relation to the other units. We can discover that a great deal of DEA models take the organizational setting into account. Cook et al. [36] framed an efficiency analysis model for a hierarchical condition where the measured units operate under the umbrella of different groups. The groups are assumed to have the power to control a part of the resources, which affects the possibilities of the assessed efficiency of the individual units. Now we will also use the DEA model to take the organizational setting into account, where the setting is similar to the above-mentioned paper that considers resource allocation and target setting problem. However, previous papers assume that the unit can reach any point on the efficiency frontier. Because of the managerial, political, and environmental limitations and so on the unit modifies its current position in the planning period to project the efficiency frontier, which is a mathematical feasibility instead of a managerial feasibility. If the central decision-maker does not emphasize the importance to consider the limitations of change in the unit's production during the planning period, he will make erroneous decisions. Like this, it will cause the unreasonableness of resource allocation and bring an excessive target which the unit cannot achieve. Moreover, it loses incentive function by allocating resource and target setting.

In this side, our approach clearly deviates from earlier papers which use the DEA model to solve the resource allocation and target setting problem. We will take the potential limitations of changing the unit's production into account to guarantee managerial feasibility by projecting onto efficiency frontier during the planning period. In general, there are a lot of influence factors which restrict the change and thus subtract a proportion of the potential output. There is no doubt that we could set a "price" for the change. But 
there are factors which cannot use the price to measure the restriction of change, such as management efforts. We will use the difficulty coefficient to weigh the limitations of reaching the efficiency frontier. During the planning period, the central decision-maker will set the difficulty coefficient of reducing centesimal input and increasing centesimal output based on the managerial experience, the limit of budget, and manager's preference. The central decider could use the analytic hierarchy process (AHP) and group decision to set the difficulty coefficient matrix. With larger value of the difficulty coefficient, it is more difficult to change the current situation of the production in the next prior.

In this section, we will propose three objectives, namely, maximizing aggregated output, minimizing the consumption of input resources, and minimizing the total difficulty coefficient, and create a model with these objectives for producing a resource allocation and target setting scheme.

3.1. Notation and Objection Functions. Before formulating the models, the required notation needs to be introduced. Here we summarize these mathematical notations as follows.

\section{Parameters}

$n$ : the number of DMUs

$k, j=1, \ldots, n$ : index for DMUs

$m$ : the number of inputs

$i=1,2, \ldots, m$ : index for inputs

$s$ : the number of outputs

$r=1,2, \ldots, s$ : index for outputs

$x_{i j}$ : the value of input $i$ consumed by DMU $j$

$y_{r j}$ : the value of output $r$ produced by DMU $j$

$\left(c_{1}, c_{2}, \ldots, c_{m}\right)$ : the difficulty coefficient vector of input reducing by projecting on the efficiency frontier

$\left(d_{1}, d_{2}, \ldots, d_{s}\right)$ : the difficulty coefficient vector of output increasing by projecting on the efficiency frontier

\section{Variables}

$\left(\lambda_{1 j}, \lambda_{2 j}, \ldots, \lambda_{n j}\right)$ : vector of variables of linear combination coefficients for DMU $j$

$\bar{x}_{i j}$ : an unknown amount of input $i$ allocated to DMU $j$

$\bar{y}_{r j}$ : an unknown amount of output $r$ targeted for DMU $j$

According to the efficiency objective, it seeks to achieve "more-for-less"; in other words, if the resources are allocated, the amount of the total outputs of the DMUs should be maximized, or the target plan of output produced is given; the resources of all the DMUs consumed will be minimized. Also we will emphasize that it is very important to take potential limitations of the change during the planning time into account.

To illustrate resource allocation and target setting, we will consider the situations based on the following general constraints.
(1) The targeted outputs shall not decrease for any of the DMUs while the unit is able to reach any point on the efficient frontier. This idea is modelled as

$$
\bar{y}_{r j} \geq y_{r j}, \quad r=1, \ldots, s ; j=1, \ldots, n .
$$

(2) The allocated inputs shall not increase for any of the DMUs while the unit is able to reach any point on the efficient frontier. Similarly, we have

$$
\bar{x}_{i j} \leq x_{i j}, \quad i=1, \ldots, m ; j=1, \ldots, n .
$$

The multiobjectives we impose are as follows.

(1) Maximizing the total output of the all DMUs:

$$
O_{1} \equiv \max \frac{1}{s} \sum_{r=1}^{s} \frac{\sum_{j=1}^{n} \bar{y}_{r j}}{\sum_{j=1}^{n} y_{r j}}
$$

(2) Minimizing the total input of the all DMUs:

$$
O_{2} \equiv \min \frac{1}{m} \sum_{i=1}^{m} \frac{\sum_{j=1}^{n} \bar{x}_{i j}}{\sum_{j=1}^{n} x_{i j}} .
$$

(3) Minimizing the total difficulty coefficient of the all DMUs:

$$
\mathrm{O}_{3} \equiv \min \frac{1}{n} \sum_{j=1}^{n}\left(\sum_{i=1}^{m} c_{i} \frac{x_{i j}-\bar{x}_{i j}}{x_{i j}}+\sum_{r=1}^{s} d_{r} \frac{\bar{y}_{r j}-y_{r j}}{y_{r j}}\right) .
$$

Thus, the first objective function is to maximize the average percentage increase in the total outputs when the resource variable of each DMU is given. The second objective function is to minimize the average percentage decrease in the total inputs when the target of each unit is set. The third objective function is to minimize the total difficulty coefficient; in other words, it is to minimize the cost of change of the current production in the next period. Also it is to engage the managerial feasibility and coincidence of the company's actual production. Traditionally, we maximize the total outputs and minimize the total inputs as the objective function. However, when the inputs or the outputs are more than one, the problem is a multiple-criteria problem. Now we choose the proportion form which has an interesting feature of the three objective functions which is invariance to the unit measurement. If any input variable or output variable changes its units of assessment, the objective function values do not change for any feasible solution. Because of the dimensionless quality of the ratios that are summed in each of the expressions, the optimal solutions of the proposed models will not change with changes in the units of assessment.

3.2. Resource Allocation and Target Setting Model. Based on the given some objective functions and some general constraints, we form a multiple objective model for allocating resource and setting target for each DMU. Let $\alpha_{i}(i=$ $1, \ldots, m)$ be the reduction factor of the total input $i, \beta_{r}(r=$ $1, \ldots, s)$ the increase factor of the total output $r$, and $t_{j}(j=$ 
$1, \ldots, n)$ the difficulty coefficient of changing the current production for $\mathrm{DMU}_{j}$. The multiple objective function is formed as follows:

$$
\begin{array}{ll} 
& \left\{\max \frac{1}{s} \sum_{r=1}^{s} \beta_{r}, \min \frac{1}{m} \sum_{i=1}^{m} \alpha_{i}, \min \frac{1}{n} \sum_{j=1}^{n} t_{j}\right\} \\
\text { s.t. } & \sum_{j=1}^{n} \bar{x}_{i j}=\alpha_{i} \sum_{j=1}^{n} x_{i j}, \quad i=1, \ldots, m \\
& \sum_{j=1}^{n} \bar{y}_{r j}=\beta_{r} \sum_{j=1}^{n} y_{r j}, \quad r=1, \ldots, s \\
& \sum_{k=1}^{n} \lambda_{k j} x_{i k} \leq \bar{x}_{i j}, \quad i=1, \ldots, m ; j=1, \ldots, n \\
& \sum_{k=1}^{n} \lambda_{k j} y_{r k} \geq \bar{y}_{r j}, \quad r=1, \ldots, s ; j=1, \ldots, n \\
& \bar{x}_{i j} \leq x_{i j}, \quad i=1, \ldots, m ; j=1, \ldots, n \\
& \bar{y}_{r j} \geq y_{r j}, \quad r=1, \ldots, s ; j=1, \ldots, n \\
t_{j}=\sum_{i=1}^{m} c_{i} \frac{x_{i j}-\bar{x}_{i j}}{x_{i j}}+\sum_{r=1}^{s} d_{r} \frac{\bar{y}_{r j}-y_{r j}}{y_{r j}}, \quad j=1, \ldots, n \\
\bar{x}_{i j}, \bar{y}_{r j}, \lambda_{k j} \geq 0, \quad{ }_{i=1} \ldots, m ; r=1, \ldots, s ; k, j=1, \ldots, n .
\end{array}
$$

Model (9) is a multiple objective linear programming. The purpose of the model is to find a feasible linear combination of the input and output vectors of each DMU that simultaneously maximizes all the outputs and minimizes all the inputs under considering potential limitations of the change in the current production during the next period. The problem, like the other multiple-criteria models, has no unique solution in general. Theoretically, any one dominant solution is reasonable in the multiple-criteria decision-making (MCDM) literature.

As to the multiple objective linear programming of model (9), we can use any MOLP technique (for more information, see, e.g., [37]) to solve the multiple objective optimization problem. A possible and currently popular way is to search for solutions of the MOLP problem on the efficient frontier. Using the production possibility set as the set of feasible operation points of each DMU, we can project any given points on the efficient frontier in the objective space. So we will use this method to search the most preferred solution under considering the potential limitations of projecting on the efficient frontier. Because of those limitations, some DMU cannot reach any point on the efficient frontier during the planning period. We must make trade-off between the maximum outputs or the minimal inputs with the total difficulty coefficient, considering the decision-maker can adjust the total difficulty coefficient based on the company's strategy. If the company's strategy is short term, he may accept a smaller difficulty coefficient. He does not want to change the current production conditions during the next term. Especially in the short run, changing the production of projecting on the efficient frontier can be very expensive. There may be labor contracts, buildings, and so on. which make it impossible to move production. So the decision-makers allocate resource and set target for each DMU considering their abilities in the actual production environment. If the company's strategy is long term, he may accept a larger difficulty coefficient. He will make the appropriate investment to change the current production for projecting onto the efficient frontier as soon as possible. For example, he can introduce the advanced production technology to improve the efficiency. The larger the difficulty coefficient the company can receive, the more the outputs that can be acquired and the more the inputs that can be saved. In other words, the decision-maker is willing to spend a total cost on moving the current production onto efficiency frontier; he would have such an efficiencygain which means achieving the maximum outputs and the minimal inputs.

When we take the total difficulty coefficient of changing the production situation which the company can accept into account, we will first fix the level of DMU's difficulty coefficient; then we will adjust the difficulty coefficient which the company can accept to observe the change of the total output increased or total input saved. The priorities of the total maximum output and the total minimal input can be done by the user. In this paper we will consider two alternations. In the first alternative, the priority is that $O_{1}$ is considered before $\mathrm{O}_{2}$; that is, firstly, the total production is maximized subject to total inputs limits; secondly, the total inputs are minimized subject to maintaining maximum total outputs. In the second alternative, the priorities are $\mathrm{O}_{2}$ before $\mathrm{O}_{1}$; that is, firstly, the total inputs are minimized subject to the total outputs limits; secondly, the total outputs are maximized subject to maintaining minimum total inputs. Also the total difficulty coefficient of changing the production situation which the company can accept is considered in the two alternatives.

\section{(1) Alternative 1}

Phase 1

$$
\begin{array}{ll}
\max & \frac{1}{s} \sum_{r=1}^{s} \beta_{r} \\
\text { s.t. } & \sum_{j=1}^{n} \bar{y}_{r j}=\beta_{r} \sum_{j=1}^{n} y_{r j}, \quad r=1, \ldots, s \\
& \sum_{k=1}^{n} \lambda_{k j} x_{i k} \leq \bar{x}_{i j}, \quad i=1, \ldots, m ; j=1, \ldots, n \\
& \sum_{k=1}^{n} \lambda_{k j} y_{r k} \geq \bar{y}_{r j}, \quad r=1, \ldots, s ; j=1, \ldots, n \\
& \bar{x}_{i j} \leq x_{i j}, \quad i=1, \ldots, m ; j=1, \ldots, n \\
& \bar{y}_{r j} \geq y_{r j}, \quad r=1, \ldots, s ; j=1, \ldots, n
\end{array}
$$




$$
\begin{aligned}
& \sum_{i=1}^{m} c_{i} \frac{x_{i j}-\bar{x}_{i j}}{x_{i j}}+\sum_{r=1}^{s} d_{r} \frac{\bar{y}_{r j}-y_{r j}}{y_{r j}} \leq t_{j}, \\
& j=1, \ldots, n \\
& \bar{x}_{i j}, \bar{y}_{r j}, \lambda_{k j} \geq 0, \\
& \quad i=1, \ldots, m ; r=1, \ldots, s ; k, j=1, \ldots, n .
\end{aligned}
$$

Model (10) seeks to maximize the total output of the all DMU subject to the following: (1) no DMU loses outputs; (2) no DMU can increase its inputs; and (3) no DMU can exceed the difficulty coefficient which the company can accept.

Let $y_{r j}^{*}$ be the optimal value of the output targets of $\mathrm{DMU}_{j}(j=1, \ldots, n)$ in model $(10)$ and $\beta_{r}^{*}$ the corresponding increase factor for output $r$ in the optimal solution of model (10). Note that the current situation represents a feasible solution in model (10) and it has an objective function solution equal to unity. Therefore, the optimal value will be greater than one (or at least equal to unity).

\section{Phase 2}

$$
\begin{array}{ll}
\min & \frac{1}{m} \sum_{i=1}^{m} \alpha_{i} \\
\text { s.t. } \quad & \sum_{j=1}^{n} \bar{x}_{i j}=\alpha_{i} \sum_{j=1}^{n} x_{i j}, \quad i=1, \ldots, m \\
& \sum_{k=1}^{n} \lambda_{k j} x_{i k} \leq \bar{x}_{i j}, \quad i=1, \ldots, m ; j=1, \ldots, n \\
& \sum_{k=1}^{n} \lambda_{k j} y_{r k} \geq \bar{y}_{r j}, \quad r=1, \ldots, s ; j=1, \ldots, n \\
& \sum_{j=1}^{n} \bar{y}_{r j} \geq \beta_{r}^{*} \sum_{j=1}^{n} \bar{y}_{r j}, \quad r=1, \ldots, s \\
& \bar{x}_{i j} \leq x_{i j}, \quad i=1, \ldots, m ; j=1, \ldots, n \\
& \bar{y}_{r j} \geq y_{r j}, \quad r=1, \ldots, s ; j=1, \ldots, n \\
& \sum_{i=1}^{m} c_{i} \frac{x_{i j}-\bar{x}_{i j}}{x_{i j}}+\sum_{r=1}^{s} d_{r j} \frac{\bar{y}_{r j}-y_{r j}}{y_{r j}} \leq t_{j}, \\
& \bar{x}_{i j}, \bar{y}_{r j}, \lambda_{k j} \geq 0, \quad j=1, \ldots, n \\
& i=1, \ldots, m ; r=1, \ldots, s ; k, j=1, \ldots, n .
\end{array}
$$

Model (11) wants to minimize the total input of the all DMU subject to the following: (1) no DMU loses outputs; (2) no DMU can increase its inputs; (3) the total output target is no less than that determined in phase 1 ; and also (4) no DMU can exceed the difficulty coefficient which the company can accept.

\section{(2) Alternative 2}

Phase 1

$$
\begin{array}{ll}
\min & \frac{1}{m} \sum_{i=1}^{m} \alpha_{i} \\
\text { s.t. } & \sum_{j=1}^{n} \bar{x}_{i j}=\alpha_{i} \sum_{j=1}^{n} x_{i j}, \quad i=1, \ldots, m \\
& \sum_{k=1}^{n} \lambda_{k j} x_{i k} \leq \bar{x}_{i j}, \quad i=1, \ldots, m ; j=1, \ldots, n \\
& \sum_{k=1}^{n} \lambda_{k j} y_{r k} \geq \bar{y}_{r j}, \quad r=1, \ldots, s ; j=1, \ldots, n \\
& \bar{x}_{i j} \leq x_{i j}, \quad i=1, \ldots, m ; j=1, \ldots, n \\
& \bar{y}_{r j} \geq y_{r j}, \quad r=1, \ldots, s ; j=1, \ldots, n \\
& \sum_{i=1}^{m} c_{i} \frac{x_{i j}-\bar{x}_{i j}}{x_{i j}}+\sum_{r=1}^{s} d_{r} \frac{\bar{y}_{r j}-y_{r j}}{y_{r j}} \leq t_{j}, \\
& \bar{x}_{i j}, \bar{y}_{r j}, \lambda_{k j} \geq 0, \quad j=1, \ldots, n \\
i=1, \ldots, m ; r=1, \ldots, s ; k, j=1, \ldots, n .
\end{array}
$$

Phase 2

$$
\begin{array}{ll}
\max & \frac{1}{s} \sum_{r=1}^{s} \beta_{r} \\
\text { s.t. } \quad & \sum_{j=1}^{n} \bar{y}_{r j}=\beta_{r} \sum_{j=1}^{n} y_{r j}, \quad r=1, \ldots, s \\
& \sum_{k=1}^{n} \lambda_{k j} x_{i k} \leq \bar{x}_{i j}, \quad i=1, \ldots, m ; j=1, \ldots, n \\
& \sum_{k=1}^{n} \lambda_{k j} y_{r k} \geq \bar{y}_{r j}, \quad r=1, \ldots, s ; j=1, \ldots, n \\
& \sum_{j=1}^{n} \bar{x}_{i j} \leq \alpha_{i}^{*} \sum_{j=1}^{n} x_{i j}, \quad i=1, \ldots, m \\
& \bar{x}_{i j} \leq x_{i j}, \quad i=1, \ldots, m ; j=1, \ldots, n \\
& \bar{y}_{r j} \geq y_{r j}, \quad r=1, \ldots, s ; j=1, \ldots, n \\
& \sum_{i=1}^{m} c_{i} \frac{x_{i j}-\bar{x}_{i j}}{x_{i j}}+\sum_{r=1}^{s} d_{r} \frac{\bar{y}_{r j}-y_{r j}}{y_{r j}} \leq t_{j}, \\
& \bar{x}_{i j}, \bar{y}_{r j}, \lambda_{k j} \geq 0, \quad j=1, \ldots, n \\
i=1, \ldots, r=1, \ldots, s ; k, j=1, \ldots, n,
\end{array}
$$


TABLE 1: Data for the illustration example.

\begin{tabular}{|c|c|c|c|c|c|}
\hline \multirow{2}{*}{ DMU } & \multicolumn{2}{|c|}{ Inputs } & \multicolumn{2}{|c|}{ Outputs } & \multirow{2}{*}{ CCR efficiency } \\
\hline & Fixed asset $\left(10^{4}\right.$ Yuan $)$ & Operating cost $\left(10^{4}\right.$ Yuan $)$ & Occupancy rate (\%) & Turnover $\left(10^{4}\right.$ Yuan $)$ & \\
\hline 1 & 245.00 & 284.00 & 31.27 & 204.00 & 0.7874 \\
\hline 2 & 384.00 & 304.00 & 38.22 & 624.00 & 1.0000 \\
\hline 3 & 229.00 & 310.00 & 27.58 & 324.00 & 0.8134 \\
\hline 4 & 272.00 & 311.00 & 44.09 & 410.00 & 1.0000 \\
\hline 5 & 314.00 & 334.00 & 40.89 & 630.00 & 1.0000 \\
\hline 6 & 266.00 & 393.00 & 37.51 & 494.00 & 1.0000 \\
\hline 7 & 379.00 & 493.00 & 42.85 & 794.00 & 1.0000 \\
\hline 8 & 565.00 & 504.00 & 56.54 & 569.00 & 0.8023 \\
\hline 9 & 625.00 & 567.00 & 57.14 & 582.00 & 0.7223 \\
\hline 10 & 624.00 & 627.00 & 86.30 & 884.00 & 0.9875 \\
\hline 11 & 623.00 & 640.00 & 51.77 & 574.00 & 0.5899 \\
\hline 12 & 599.00 & 647.00 & 91.50 & 769.00 & 0.9976 \\
\hline 13 & 583.00 & 698.00 & 81.60 & 600.00 & 0.8635 \\
\hline 14 & 668.00 & 722.00 & 83.20 & 869.00 & 0.8322 \\
\hline 15 & 754.00 & 773.00 & 54.63 & 838.00 & 0.5733 \\
\hline 16 & 671.00 & 776.00 & 84.40 & 756.00 & 0.7760 \\
\hline Average & 487.56 & 523.94 & 56.84 & 620.06 & 0.8591 \\
\hline
\end{tabular}

where $\alpha_{i}^{*}$ is the optimal value of the input decreased factor obtained in model (12).

In addition to the proposed approach being the feature of unit invariant, obviously, an additional important feature is its flexibility. Thus, we can give a different relative weight which can be reflected in decision-maker's subjective preference for different outputs, inputs, and difficulty coefficients. Assuming a weight $w_{r}(r=1, \ldots, s)$ for output $r$ to maximize them, it would only be needed to substitute the objective functions in model (9) to (13) by $\max (1 / s) \sum_{r=1}^{s} w_{r} \beta_{r}$. Similarly, a different, relative weight $\delta_{i}(i=1, \ldots, m)$ can be given to input $i$ to minimize them. So we will modify the objective functions in model (9) and (13) by $\min (1 / m) \sum_{i=1}^{m} \delta_{i} \alpha_{i}$. Also a different, relative weight $\xi_{j}(\xi=1, \ldots, n)$ can be given to DMU $j$ to minimize them. So we will modify the objective functions in model (9) and (13) by $\min (1 / n) \sum_{j=1}^{n} \xi_{j} t_{j}$.

\section{Numerical Example}

In this section, the advanced characteristic and practical values in our proposed model are demonstrated. To this end, we analyzed resource allocation and target setting simultaneously based on 2012 annual report of 16 sets of chain hotels located in Anhui, China. These hotels belong to the same chain with single central decision-making team to supervise all their branches. The company consists of 16 branches (DMUs), each of which uses two inputs and two outputs. The two output variables are room occupancy rate and turnover. Room occupancy rate is an index of hotel popularity defined by the ratio of number of occupied rooms to total number of guest rooms for the hotel business reference analysis. Turnover profits from room revenue, food and beverage income, and other income. On the other hand, two input variables are fixed assets and operating costs. Fixed assets are from facilities and equipment and other supplies that are used for hotel operations, while rent, staff salaries, office expenses, material consumption, and other expenses form operating costs. The raw data of the example studied in this paper is shown in Table 1.

First of all, we can use model (2) to calculate the classic CCR efficiency scores for these 16 hotels. The results are listed in the last column of Table 1 . As can be seen, five hotels (2. 4, 5, 6 , and 7) are efficient with an efficiency score of one, implying that these hotels are operating very well in the year of 2012 as compared with their peers. On the contrary, the remaining eleven hotels are inefficient, and the lowest efficiency score appears for 15th hotel reaching 0.5733 . For these hotels as a whole, the average efficiency score reaches 0.8591 , which shows that these chain hotels have realized a relative high performance.

Then, we solve model (10)-(13) and obtain the optimization solutions without taking potential limitations of changing the current production into account. Table 2 shows the optimal values of resource allocation and target setting of each DMU in alternative 1. This achieves the total maximum outputs which have precedence over the total minimum input. From Table 2, we can find the average occupancy rate increases from $56.84 \%$ to $62.85 \%$ and the average turnover increases from 620.06 to 892.92 . Also we can find every output of each DMU is no less than the raw data in the existing production. After allocating resource and setting output target, we will find all DMU projecting onto the efficient frontier. $\mathrm{DMU}_{2}, \mathrm{DMU}_{4}, \mathrm{DMU}_{5}, \mathrm{DMU}_{6}$, and $\mathrm{DMU}_{7}$ keep unchanged after resource allocation and target setting.

When we will pursue the total minimum input which has precedence over the total maximum outputs. We will use alternative 2 and solve model (12) and model (13) to obtain the optimization solutions. The outcome of alternative 2 will be given in Table 3. From Table 3, we can find the 
TABLE 2: Resource allocation and target setting for alternative 1.

\begin{tabular}{|c|c|c|c|c|c|}
\hline $\mathrm{DMU}$ & Fixed asset & Operating cost & Occupancy rate & Turnover & Efficiency \\
\hline 1 & 245.00 & 260.61 & 31.90 & 491.56 & 1.0000 \\
\hline 2 & 384.00 & 304.00 & 38.22 & 624.00 & 1.0000 \\
\hline 3 & 229.00 & 243.59 & 29.82 & 459.46 & 1.0000 \\
\hline 4 & 272.00 & 311.00 & 44.09 & 410.00 & 1.0000 \\
\hline 5 & 314.00 & 334.00 & 40.89 & 630.00 & 1.0000 \\
\hline 6 & 266.00 & 393.00 & 37.51 & 494.00 & 1.0000 \\
\hline 7 & 379.00 & 493.00 & 42.85 & 794.00 & 1.0000 \\
\hline 8 & 565.00 & 504.00 & 62.63 & 997.63 & 1.0000 \\
\hline 9 & 625.00 & 567.00 & 70.35 & 1116.86 & 1.0000 \\
\hline 10 & 624.00 & 627.00 & 86.30 & 945.07 & 1.0000 \\
\hline 11 & 623.00 & 640.00 & 78.57 & 1218.17 & 1.0000 \\
\hline 12 & 599.00 & 647.00 & 91.50 & 863.22 & 1.0000 \\
\hline 13 & 583.00 & 698.00 & 81.60 & 1084.03 & 1.0000 \\
\hline 14 & 668.00 & 710.55 & 86.99 & 1340.25 & 1.0000 \\
\hline 15 & 754.00 & 773.00 & 94.91 & 1472.11 & 1.0000 \\
\hline 16 & 671.00 & 713.74 & 87.38 & 1346.27 & 1.0000 \\
\hline Average & 487.56 & 513.72 & 62.85 & 892.92 & 1.0000 \\
\hline
\end{tabular}

TABLE 3: Resource allocation and target setting for alternative 2.

\begin{tabular}{|c|c|c|c|c|c|}
\hline$\overline{\mathrm{DMU}}$ & Fixed asset & Operating cost & Occupancy rate & Turnover & Efficiency \\
\hline 1 & 192.91 & 220.57 & 31.27 & 204.00 & 1.0000 \\
\hline 2 & 384.00 & 304.00 & 38.22 & 624.00 & 1.0000 \\
\hline 3 & 186.84 & 206.86 & 27.58 & 324.00 & 1.0000 \\
\hline 4 & 272.00 & 311.00 & 44.09 & 410.00 & 1.0000 \\
\hline 5 & 314.00 & 334.00 & 40.89 & 630.00 & 1.0000 \\
\hline 6 & 266.00 & 393.00 & 37.51 & 494.00 & 1.0000 \\
\hline 7 & 379.00 & 493.00 & 42.85 & 794.00 & 1.0000 \\
\hline 8 & 359.49 & 406.71 & 56.54 & 569.00 & 1.0000 \\
\hline 9 & 365.03 & 412.29 & 57.14 & 582.00 & 1.0000 \\
\hline 10 & 552.54 & 623.61 & 86.30 & 884.00 & 1.0000 \\
\hline 11 & 342.27 & 382.07 & 51.77 & 574.00 & 1.0000 \\
\hline 12 & 564.48 & 645.42 & 91.50 & 769.00 & 1.0000 \\
\hline 13 & 503.41 & 575.59 & 81.60 & 600.00 & 1.0000 \\
\hline 14 & 536.84 & 604.26 & 83.20 & 869.00 & 1.0000 \\
\hline 15 & 418.60 & 445.56 & 54.63 & 838.00 & 1.0000 \\
\hline 16 & 520.68 & 595.34 & 84.40 & 756.00 & 1.0000 \\
\hline Average & 384.88 & 434.58 & 56.84 & 620.10 & 1.0000 \\
\hline
\end{tabular}

average fixed asset decreases from 487.56 to 384.88 and the average operating cost decreases from 523.94 to 434.58 . Also we can find that every input of each adjusted DMU is no more than the original inputs. After allocating resource and setting output target, we will find again that all DMU are fully efficient, which is the same as that of alternative 1.

Then we will consider potential limitations of changing the current production. For simplicity and without loss of generality, we will analyze alternative 1 . After allocating resource and setting output target, we will find all DMU projecting the efficient frontier. This is impossible to achieve for the next term's production planning. If the decision-makers do not pay attention to the potential limitations and their abilities of changing the current production condition, they will make some incorrect decisions. So we want to take the problems of restrictions on projecting onto the efficiency frontier which might be very expensive into account. The decision-makers define the difficulty coefficient based on the company's strategy. If the company can accept the total difficulty coefficient, the company will reach the efficient frontier easily in the next term. Also, the company can save the total input and gain more total output. Now the company identifies the difficulty coefficient matrix which is from the current production to next production condition. 


\section{Conclusion}

DEA has been traditionally used for measuring the performance of individual units. Currently, DEA is also a useful approach for solving the problem of resource allocation and target setting popularly, since Lozano and Villa [25] approached such problem by introducing the concept of centralized data envelopment analysis (CDEA) models to minimize the overall resource consumption or to maximize the over output production in an organization, rather than considering each DMU separately. There are some literatures considering the CDEA. In these literatures, they are mainly based on the empirical characterization of production possibility set formed by DEA and projecting the inefficient unit onto the efficient frontier. However, only few works consider achieving or not. This is because the efficient frontier only gives a direction of changing the current production condition during the next term. In this paper, we want to take the limits into account and form a difficulty coefficient matrix of changing the production in the resource allocation and target setting. So as to do that, we want to guarantee managerial feasibility rather than mathematical feasibility in the planning period. So we presented a DEA-based approach for resource allocation and target setting problem. It involves three objectives, namely, output maximization, total resources consumption minimization, and the total minimum accepted difficulty coefficient. This is a multiobjective linear programming. We use the lexicographic order in which these objectives are purposed. Because the accepted difficulty coefficient of each unit is based on the decisionmaker preference and the company's strategy, we investigate the relationship between the total difficulty coefficient and the maximum outputs and minimum inputs. By doing that, the managers of organizations are willing to put in changing the production condition and managerial style and expect the total output increased and the total input saved, that is, the efficiency-gain associated with this resource allocation and target setting. Having such relationship, the managers can choose to stop investing into input allocation and output setting when an accepted level of total output increased and input saved is reached.

In this paper we use the CCR model; we also can extend the approach to other DEA models. For instance, we can obtain the scheme of resource allocation and target setting under the condition of variable returns to scale (VRS). In addition, the inputs and outputs are only measured by exact values in our work. Future research can extend the present approach to consider the cases where the inputs and outputs of DMUs are bounded data, ordinal data, and ratiobounded data. Also, one can take the interaction among DMUs into account. By saying interaction, we mean that DMUs can cooperate, coordinate, and even negotiate towards the completion of the problem of resource allocation and target setting. That is a direction for further research where there are interdependencies between DMUs.

\section{Conflicts of Interest}

No potential conflicts of interest were reported by the authors.

\section{References}

[1] A. Charnes, W. W. Cooper, and E. Rhodes, "Measuring the efficiency of decision making units," European Journal of Operational Research, vol. 2, no. 6, pp. 429-444, 1978.

[2] A. Emrouznejad, B. R. Parker, and G. Tavares, "Evaluation of research in efficiency and productivity: a survey and analysis of the first 30 years of scholarly literature in DEA," Socio-Economic Planning Sciences, vol. 42, no. 3, pp. 151-157, 2008.

[3] F. Li, L. Liang, Y. Li, and A. Emrouznejad, "An alternative approach to decompose the potential gains from mergers," Journal of the Operational Research Society, 2018, In Press.

[4] F. Li, Q. Zhu, and J. Zhuang, "Analysis of fire protection efficiency in the United States: a two-stage DEA-based approach," OR Spectrum, pp. 1-46, 2017.

[5] W. D. Cook and M. Kress, "Characterizing an equitable allocation of shared costs: a DEA approach," European Journal of Operational Research, vol. 119, no. 3, pp. 652-661, 1999.

[6] G. R. Jahanshahloo, F. Hosseinzadeh Lotfi, N. Shoja, and M. Sanei, "An alternative approach for equitable allocation of shared costs by using DEA," Applied Mathematics and Computation, vol. 153, no. 1, pp. 267-274, 2004.

[7] G. R. Jahanshahloo, F. Hosseinzadeh Lotfi, and M. Moradi, "A DBA approach for fair allocation of common revenue," Applied Mathematics and Computation, vol. 160, no. 3, pp. 719-724, 2005.

[8] J. E. Beasley, "Allocating fixed costs and resources via data envelopment analysis," European Journal of Operational Research, vol. 147, no. 1, pp. 198-216, 2003.

[9] L. Fang and C.-Q. Zhang, "Resource allocation based on the DEA model," Journal of the Operational Research Society, vol. 59, no. 8, pp. 1136-1141, 2008.

[10] R. Lin and Z. Chen, "Fixed input allocation methods based on super CCR efficiency invariance and practical feasibility," Applied Mathematical Modelling, vol. 40, no. 9-10, pp. 53775392, 2016.

[11] M.-M. Yu, L.-H. Chen, and B. Hsiao, "A fixed cost allocation based on the two-stage network data envelopment approach," Journal of Business Research, vol. 69, no. 5, pp. 1817-1822, 2016.

[12] F. Li, Q. Zhu, and L. Liang, "Allocating a fixed cost based on a DEA-game cross efficiency approach," Expert Systems with Applications, vol. 96, pp. 196-207, 2018.

[13] B. Golany, "An interactive MOLP procedure for the extension of DEA to effectiveness analysis," Journal of the Operational Research Society, vol. 39, no. 8, pp. 725-734, 1988.

[14] A. D. Athanassopoulos, "Goal programming \& data envelopment analysis (GoDEA) for target-based multi-level planning: allocating central grants to the Greek local authorities," European Journal of Operational Research, vol. 87, no. 3, pp. 535-550, 1995.

[15] A. D. Athanassopoulos, "Decision support for target-based resource allocation of public services in multiunit and multilevel systems," Management Science, vol. 44, no. 2, pp. 173-187, 1998.

[16] R. K. Matin and R. Azizi, "A two-phase approach for setting targets in DEA with negative data," Applied Mathematical Modelling, vol. 35, no. 12, pp. 5794-5803, 2011.

[17] A. Dehnokhalaji, M. Ghiyasi, and P. Korhonen, "Resource allocation based on cost efficiency," Journal of the Operational Research Society, vol. 68, no. 10, pp. 1279-1289, 2017. 
[18] A. Amirteimoori and S. Kordrostami, "Allocating fixed costs and target setting: a DEA-based approach," Applied Mathematics and Computation, vol. 171, no. 1, pp. 136-151, 2005.

[19] A. Amirteimoori and M. M. Tabar, "Resource allocation and target setting in data envelopment analysis," Expert Systems with Applications, vol. 37, no. 4, pp. 3036-3039, 2010.

[20] G. Bi, J. Ding, Y. Luo, and L. Liang, "Resource allocation and target setting for parallel production system based on DEA," Applied Mathematical Modelling, vol. 35, no. 9, pp. 4270-4280, 2011.

[21] R. Lin, "Allocating fixed costs or resources and setting targets via data envelopment analysis," Applied Mathematics and Computation, vol. 217, no. 13, pp. 6349-6358, 2011.

[22] F. H. Lotfi, A. Hatami-Marbini, P. J. Agrell, N. Aghayi, and K. Gholami, "Allocating fixed resources and setting targets using a common-weights DEA approach," Computers \& Industrial Engineering, vol. 64, no. 2, pp. 631-640, 2013.

[23] A. Hatami-Marbini, M. Tavana, P. J. Agrell, F. Hosseinzadeh Lotfi, and Z. Ghelej Beigi, "A common-weights DEA model for centralized resource reduction and target setting," Computers \& Industrial Engineering, vol. 79, pp. 195-203, 2015.

[24] F. Li, J. Song, A. Dolgui, and L. Liang, "Using common weights and efficiency invariance principles for resource allocation and target setting," International Journal of Production Research, vol. 55, no. 17, pp. 4982-4997, 2017.

[25] S. Lozano and G. Villa, "Centralized resource allocation using data envelopment analysis," Journal of Productivity Analysis, vol. 22, no. 1-2, pp. 143-161, 2004.

[26] S. Lozano and G. Villa, "Centralized DEA models with the possibility of downsizing," Journal of the Operational Research Society, vol. 56, no. 4, pp. 357-364, 2005.

[27] S. Lozano, G. Villa, and B. Adenso-Díaz, "Centralised target setting for regional recycling operations using DEA," Omega , vol. 32, no. 2, pp. 101-110, 2004.

[28] M. Asmild, J. C. Paradi, and J. T. Pastor, "Centralized resource allocation BCC models," Omega, vol. 37, no. 1, pp. 40-49, 2009.

[29] P. Korhonen and M. Syrjänen, "Resource allocation based on efficiency analysis," Management Science, vol. 50, no. 8, pp.11341144, 2004.

[30] E. V. Pachkova, "Restricted reallocation of resources," European Journal of Operational Research, vol. 196, no. 3, pp. 1049-1057, 2009.

[31] S. Lozano, G. Villa, and R. Brännlund, "Centralised reallocation of emission permits using DEA," European Journal of Operational Research, vol. 193, no. 3, pp. 752-760, 2009.

[32] S. Lozano, G. Villa, and D. Canca, "Application of centralised DEA approach to capital budgeting in Spanish ports," Computers \& Industrial Engineering, vol. 60, no. 3, pp. 455-465, 2011.

[33] S. Lozano, "Nonradial approach to allocating fixed-costs and common revenue using centralized dea," International Journal of Information Technology \& Decision Making, vol. 13, no. 1, pp. 29-46, 2014.

[34] L. Fang, "Centralized resource allocation based on efficiency analysis for step-by-step improvement paths," Omega, vol. 51, pp. 24-28, 2015.

[35] L. Fang, "Centralized resource allocation DEA models based on revenue efficiency under limited information," Journal of the Operational Research Society, vol. 67, no. 7, pp. 945-952, 2016.

[36] W. D. Cook, D. Chai, J. Doyle, and R. Green, "Hierarchies and Groups in DEA," Journal of Productivity Analysis, vol. 10, no. 2, pp. 177-198, 1998.
[37] R. E. Steuer, Multiple Criteria Optimization: Theory, Computation, and Application, John Wiley \& Sons, New York, NY, USA, 1986. 


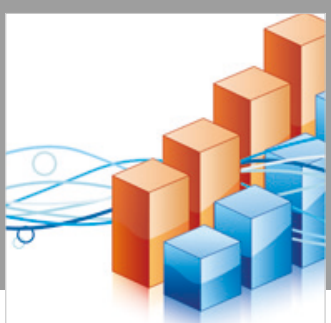

Advances in

Operations Research

\section{-n-m}
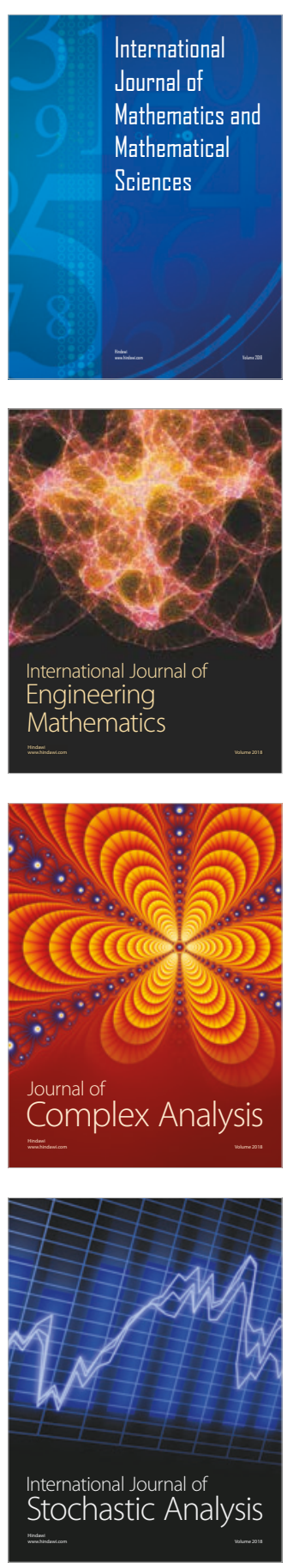
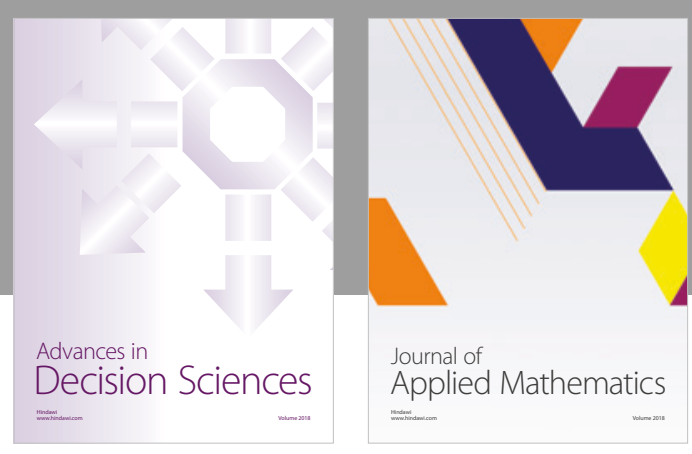

Journal of

Applied Mathematics
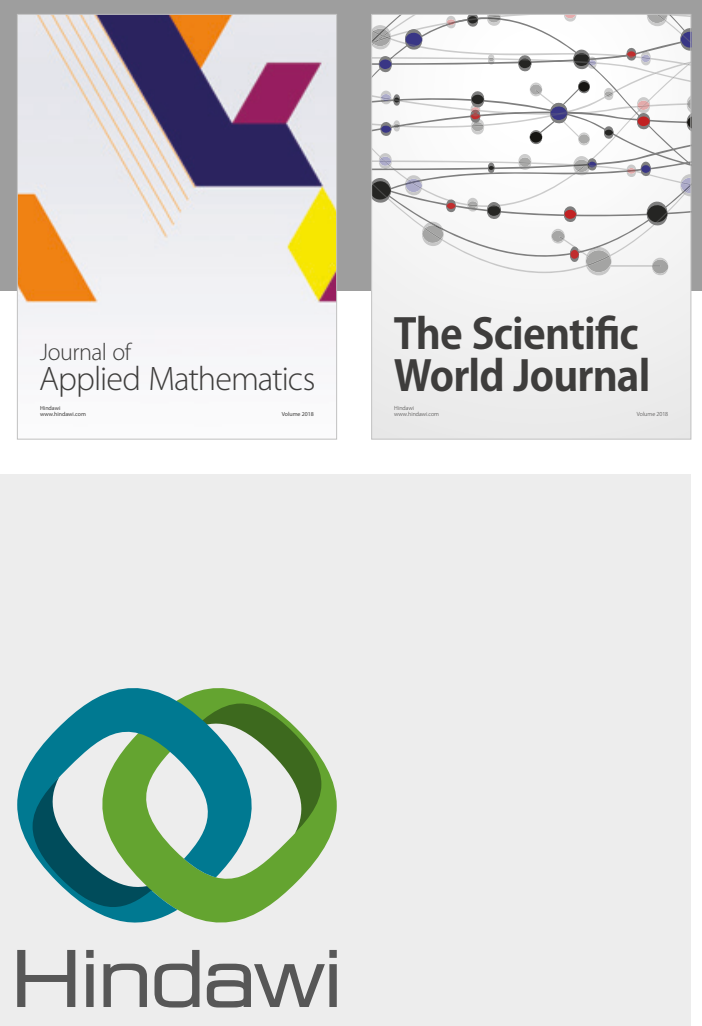

Submit your manuscripts at

www.hindawi.com

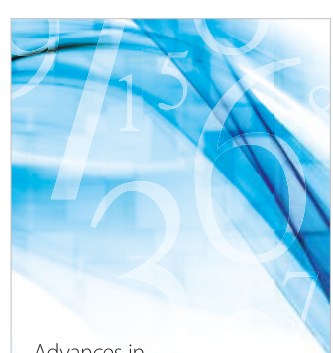

Advances in
Numerical Analysis
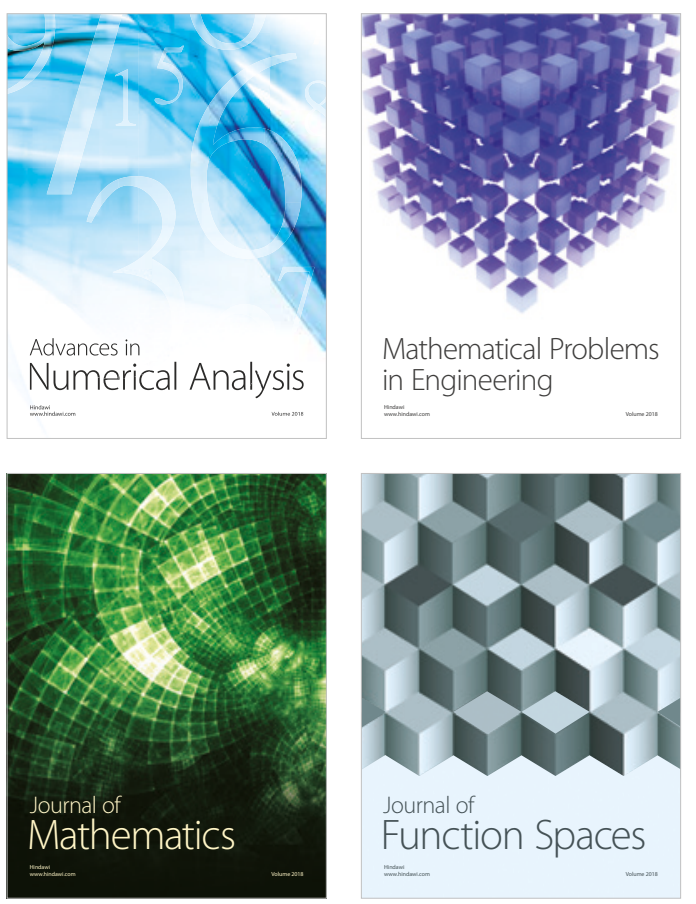

Mathematical Problems in Engineering

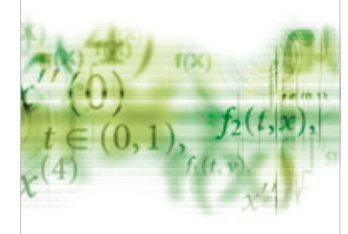

International Journal of

Differential Equations

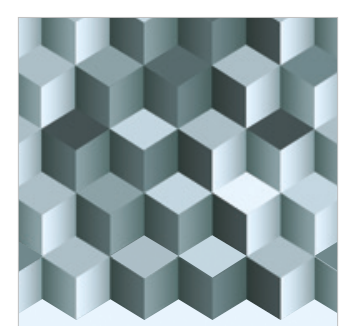

Journal of

Function Spaces

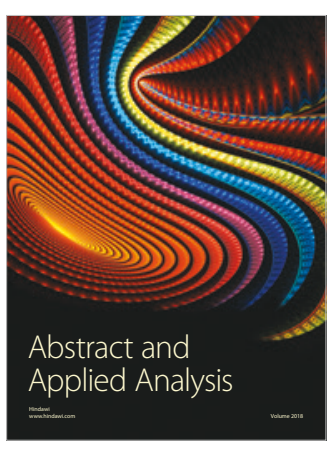

The Scientific

World Journal

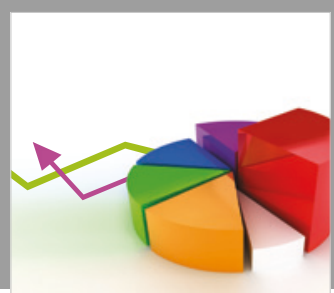

Journal of

Probability and Statistics
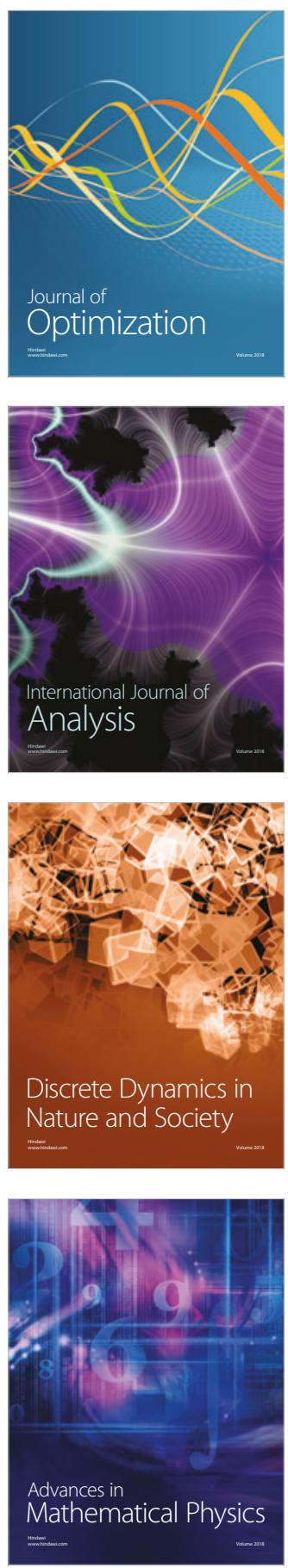